\title{
Editorial
}

Psychotherapy and Psychosomatics

Psychother Psychosom 2006;75:4

DOI: $10.1159 / 000089220$

\section{The Journal's Appreciation}

Anagnostopoulos F. (Athens, Greece)

Ananth J. (Torrance, Calif., USA)

Andreoli A. (Geneva, Switzerland)

Anton F. (Luxembourg)

Bagby M. (Toronto, Canada)

Bakker A. (Leidschendam,

The Netherlands)

Balestrieri M. (Udine, Italy)

Bankier B. (Boston, Mass., USA)

Barbui C. (Verona, Italy)

Battaglia M. (Milan, Italy)

Baune B. (Münster, Germany)

Bearss K. (New Haven, Conn., USA)

Belaise C. (Bologna, Italy)

Benazzi F. (Forlì, Italy)

Bermond B. (Amsterdam,

The Netherlands)

Berrocal C. (Malaga, Spain)

Bockting C.L.H. (Amsterdam,

The Netherlands)

Bouman T. (Groningen, The Netherlands)

Bravi A. (Bologna, Italy)

Büchi S. (Zurich, Switzerland)

Carta M. (Cagliari, Italy)

Cohen L.S. (Boston, Mass., USA)

Corazziari E. (Rome, Italy)

Costantini A. (Rome, Italy)

delle Fave A. (Milan, Italy)

De Paoli L. (Rome, Italy)

Di Serio C. (Milan, Italy)

Fabbri S. (Bologna, Italy)

Facchinetti F. (Modena, Italy)

Falloon I.R.H. (Auckland, New Zealand)

Fama J.M. (Boston, Mass., USA)

Faravelli C. (Florence, Italy)

Fassino S. (Turin, Italy)

Favaro A. (Padua, Italy)

Feiler S. (Basel, Switzerland)

Ferguson E. (Nottingham, UK)

Ferrari S. (Modena, Italy)

Flor H. (Mannheim, Germany)

Galderisi S. (Naples, Italy)
Galeazzi G.M. (Modena, Italy)

Gaudiano B. (Providence, R.I., USA)

Gelenberg A.J. (Tucson, Ariz., USA)

Ghaemi N. (Atlanta, Ga., USA)

Grabe H.J. (Stralsund, Germany)

Grace S.L.L. (Toronto, Canada)

Grassi L. (Ferrara, Italy)

Grossi G. (Stockholm, Sweden)

Guidi J. (Bologna, Italy)

Hammelstein P. (Münster, Germany)

Healy D. (Bangor, UK)

Hendin H. (New York, N.Y., USA)

Henser I. (Berlin, Germany)

Hoglend P. (Oslo, Norway)

Hoyer T. (Dresden, Germany)

Jager B. (Hannover, Germany)

Jarrett R. (Dallas, Tex., USA)

Jorgensen R.S. (Syracuse, N.Y., USA)

Kaltiala-Heino R. (Pitkaniemi, Finland)

Keitner G.I. (Providence, R.I., USA)

Keuthen M. (Charleston, Mass., USA)

Kuehner C. (Mannheim, Germany)

Labbate L. (North Little Rock, Ark., USA)

Langewitz W. (Basel, Switzerland)

Lazaratou H. (Athens, Greece)

Levenstein S. (Rome, Italy)

Linden M. (Berlin, Germany)

Linley A. (Leicester, UK)

Macleod A. (Egham, UK)

Marchesi C. (Parma, Italy)

Mataix-Cols D. (London, UK)

Maule S. (Turin, Italy)

Meyer T. (Berlin, Germany)

Meyer-Lindenberg A. (Bethesda, Md., USA)

Milos G. (Zurich, Switzerland)

Moncrieff J. (London, UK)

Monteleone P. (Naples, Italy)

Mota Cardoso R. (Porto, Portugal)

Nakagawa A. (Okayama, Japan)

Natale V. (Bologna, Italy)

Nickel M. (Simbach am Inn, Germany)
Nierenberg A. (Boston, Mass., USA)

Ottolini F. (Modena, Italy)

Pariante C. (London, UK)

Patten S.B. (Calgary, Canada)

Paykel E.S. (Cambridge, UK)

Picardi A. (Rome, Italy)

Pope H.G. (Belmont, Mass., USA)

Porcelli P. (Castellana Grotte, Italy)

Rafanelli C. (Bologna, Italy)

Remschmidt H. (Marburg, Germany)

Rigatelli M. (Modena, Italy)

Rihmer Z. (Budapest, Hungary)

Roncuzzi R. (Bologna, Italy)

Rufer M. (Zurich, Switzerland)

Rumpf H.J. (Lübeck, Germany)

Rush A.J. (Dallas, Tex., USA)

Ryan C. (Providence, R.I., USA)

Sanavio E. (Padua, Italy)

Schneider G. (Münster, Germany)

Schore A.N. (Northridge, Calif., USA)

Schwaiger U. (Lübeck, Germany)

Semprini F. (Bologna, Italy)

Sibilia L. (Rome, Italy)

Simpson G.M. (Los Angeles, Calif., USA)

Sirri L. (Bologna, Italy)

Smith R.C. (East Lansing, Mich., USA)

Söndergaard H.P. (Stockholm, Sweden)

Sonino N. (Padua, Italy)

Sorensen C.H. (Odense, Denmark)

Starcevic V. (Sydney, Australia)

Stewart D.E. (Toronto, Canada)

Swerdloff R.S. (Torrance, Calif., USA)

Takimoto Y. (Tokyo, Japan)

Tarrier N. (Manchester, UK)

Todarello O. (Bari, Italy)

Tomba E. (Boston, Mass., USA)

Tossani E. (Bologna, Italy)

Van Houdenhove B. (Leuven, Belgium)

Winkler D. (Vienna, Austria)

Wittman L. (Zurich, Switzerland)

Zachariae R. (Aarhus, Denmark)

Zimmermann C. (Verona, Italy)

\section{KARGER}

Fax +4161306 1234

E-Mail karger@karger.ch

www.karger.com
(C) $2006 \mathrm{~S}$. Karger AG, Basel

0033-3190/06/0751-0004\$23.50/0

Accessible online at:

www.karger.com/pps 